\title{
Reductive electrografting of in situ produced diazopyridinium cations: tailoring the interface between carbon electrodes and electroactive bacterial films.
}

Hassiba Smida, Estelle Lebègue, Jean-François Bergamini, Frédéric Barrière* and Corinne Lagrost*

Institut des Sciences Chimiques de Rennes-UMR 6226, CNRS-université de Rennes 1, Equipe MaCSE, Campus de Beaulieu, 35042 Rennes cedex (France)

E-mail : corinne.lagrost@univ-rennes1.fr, frederic.barriere@univ-rennes1.fr

\begin{abstract}
Carbon electrodes were functionalized through the reduction of diazopyridinium cations that are produced from in situ diazotization of 2-, 3- and 4-aminopyridine. Diazopyridinium salts were much more rarely employed for surface functionalization than other aryldiazonium derivatives. A study combining X-ray Photoelectron Spectroscopy (XPS), contact angle, ellipsometry, Atomic Force Microscopy (AFM) measurements and electrochemical analyses demonstrates that films obtained from 4-diazopyridinium cations are hydrophilic, dense, compact but sufficiently thin to preserve fast electronic transfer rate, being then relevant to efficiently tailor the interface between the anode surface and an electroactive biofilm. Microbial Fuels Cells (MFCs) with pyridine-functionalized graphite anodes exhibit faster development and improved performances than MFCs operating with bare graphite anodes.
\end{abstract}

Keywords: surface functionalization $\bullet$ diazopyridinium $\bullet$ electrochemical grafting $\bullet$ biofilm $\bullet$ microbial fuel cells

\section{Introduction}

Bioelectrochemical devices use bacteria to catalyse oxidation and/or reduction reactions at electrodes with applications in bioremediation, wastewater treatment, power production and production of chemicals with high added-value.[1] Biofilm adhesion at the anode surface and electron transfer rate at the biofilm/anode interface are key factors determining the 
performance of devices.[2] Hence, many researches are currently devoted to optimize the electrode morphology and chemistry in order to promote bacterial attachment and to facilitate electron transfer. Different strategies related to surface structuration, surface modification and/or activation were developed on carbon-based materials and have recently been reviewed.[3],[4],[5],[6],[7],[8] Among these strategies, the chemical functionalization of the bioanode through diazonium chemistry has been demonstrated to be successful at increasing the current densities, notably in Microbial Fuel Cells (MFCs).[9],[10],[11],[12],[13],[14],[15] The electron transfer from the electroactive bacteria to the anode is indeed affected by the anode properties and its surface modification. The introduction of nitrogen functionalities and/or hydrophilic properties has been suggested to improve electron transfer between electrode and electroactive bacteria.[9]'[10]'[13]'[14]'[15] Only one work uses an affinity approach with grafted boronic acid functionalities targeting the lipopolysaccharide diols of the bacteria outer-membrane.[12] Importantly, apart hydrophilic and/or affinity properties, the chemical layer should also be thin enough in order to maintain fast electron transfer kinetics between the biofilm and the electrode. The electrografting procedure with diazonium cations is nowadays recognized as a powerful and general surface modification technique that can be applied to a wide range of materials, including metals $(\mathrm{Pt}, \mathrm{Au}, \mathrm{Zn}, \mathrm{Cu}$, etc) or graphitic surfaces (graphite, glassy carbon, etc), or semiconductors ( $\mathrm{SiH}, \mathrm{SiOC}, \mathrm{SiO}_{2}$ etc). It produces a robust interface, which can be finely tuned depending on the substituents borne by the aryl unit.[16] Upon reduction, the aryl diazonium cations lead to the corresponding aryl radicals by loss of dinitrogen and these very reactive radicals are able to react with a surface. Aryl diazonium salts have been widely studied, and can be easily isolated from amine precursors or generated in situ.[16] However, due to the high reactivity of the aryl radicals, it is still difficult to form homogeneous and ultrathin layer through the control of experimental conditions (nature of solvent, concentration of diazonium cations, electrolysis time) because the radical species can attack the already-attached aryl moieties. For instance, a short electrolysis time (low charge consumption for the electrografting) generally leads to the formation of a sparse monolayer.[16],[17]

Very few works describe the use of diazopyridinium salts although they could give a direct access to the robust grafting of pyridine onto different surface. Diazopyridinium is not available as a stable salt and requires the in situ diazotization of the corresponding aminopyridine precursor. The diazotization leads to the corresponding hydroxypridine as the main products.[18] Surface modification through electrografting from diazopyridinium 
cations has been mostly carried out with 3-diazopyridinium cations,[19-23] because 4-aminoand even more 2-amino-diazopyridinium salts are considered highly unstable and claimed not to be useful for surface functionalization.[22],[21] However, some works report the grafting from 4-amino precursors.[24,25]

In this work, we report the electrografting of in situ produced diazopyridinium cations using 2-, 3- and 4-aminopyridine (Scheme 1) in order to functionalize the carbon surface with pyridine units. Pyridine molecules are known to promote rapid exchange of electrons between c-type cytochromes, including cytochrome c itself, and the electrode.[26]'[27] This is potentially interesting for tailoring the interface between the anode and the biofilm. Indeed, several microorganisms can produce electricity but some of them called exoelectrogens are especially competent to directly transfer electrons derived from the catabolism of organic matter to the anode.[28] One such genera is Geobacter which is commonly found in anodic biofilms obtained from wastewater inoculum and fed with acetate.[29] Geobacter sulfurreducens is one of the most widely studied exoelectrogen microorganism and was shown to be able to transfer electrons originating from acetate oxidation directly to the graphite electrode, without added electron-transfer mediator,[30] through outer-membrane ctype cytochromes.[31]

X-ray Photoelectron Spectroscopy (XPS) and electrochemical techniques are used to study the immobilization of pyridine units onto the surface with the different diazopyridinium isomers. The surface modification is characterized in terms of layer thickness, compactness and electron transfer blocking properties. The layer obtained with 4-aminopyridine is further selected to be implemented in MFCs and the performance of the biofuel cells is compared to MFCs using bare graphite as the anode. The pyridine units, with protruding nitrogen, are shown to be favorable for the development of the biofilm and for the performance of the bioanode in the early stage of the biofilm growth.

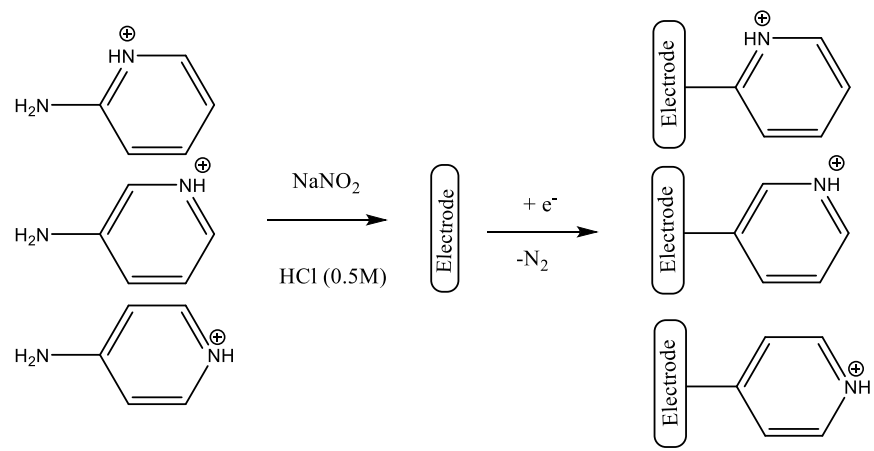


Scheme 1. Electrografting of in situ produced diazopyridinium cations onto the electrode surface. Note that multilayer polyphenylene-like modification are usually obtained as opposed to monolayers represented here for clarity only.[17]

\section{Results and Discussion}

\subsection{Reactivity of aminopyridine derivatives upon diazotization.}

2-amino-, 3-amino- and 4-aminopyridine have been shown to diazotize in diluted aqueous mineral acids.[18,32-34] After diazotization, the generated diazonium salt leads to the corresponding hydroxypridine as the main decomposition product.[18] The diazotization of the three amino derivatives was studied by UV-Visible absorption spectroscopy under the experimental conditions used for electrografting, i.e. in situ production of the corresponding diazopyridinium cations upon addition of 2 eq. $\mathrm{NaNO}_{2}$. First, spectra of the three aminopyridine compounds were recorded in $0.5 \mathrm{M} \mathrm{HCl}$ and taken as reference. They display the characteristic absorption bands of the protonated derivatives at 230 and $300 \mathrm{~nm}$ for 2aminopyridine, 250 and $315 \mathrm{~nm}$ for 3-aminopyridine and $262 \mathrm{~nm}$ for 4-aminopyridine (Figure 1).[35] After addition of 2eq. $\mathrm{NaNO}_{2}$, spectra were systematically recorded at increased time. Figure 1 shows that the spectra have different evolutions according to the nature of the protonated aminopyridine derivatives. As also reported by Agullo et al.,[20] 3-aminopyridine diazotizes and quickly forms its corresponding hydrolyzed adduct (3-hydroxypyridinium) after $60 \mathrm{~min}$ (Figure 1B). The band at $315 \mathrm{~nm}$ decreased after $1 \mathrm{~min}$ and fully disappeared after $60 \mathrm{~min}$, indicating that the 3-aminopyridinium has completely reacted. For times ranging between 1 and $10 \mathrm{~min}$, the broad band centered at $283 \mathrm{~nm}$ corresponds to the overlapping of bands due to diazopyridinium and its hydrolyzed adducts.[20]

In sharp contrast, slower evolution of the two others derivatives to hydroxypyridinium is observed. In the 2-aminopyridine spectrum (Figure 1A), the intensity of absorption band at $300 \mathrm{~nm}$ decreased along with a broadening of the band, and this behavior becomes clearly visible 60 min after the addition of $\mathrm{NaNO}_{2}$. The band broadening leads to the growth of a new band at $283 \mathrm{~nm}$, which finally blue-shifts to $278 \mathrm{~nm}$ after $24 \mathrm{~h}$ (cyan solid line in Figure 1A). This latter band corresponds to the absorption of 2-hydroxypyridinium molecules $\left(\lambda_{\max }=278\right.$ $\mathrm{nm}$, dotted line in Figure 1A). The broad band at $283 \mathrm{~nm}$ can be assigned to the mixed absorptions of the produced diazopyridinium cations [32,34] and of 2-hydroxypyridinium molecules which are formed from diazopyridinium hydrolysis. 
Similarly, after addition of $\mathrm{NaNO}_{2}$, the intensity of the absorption band of 4-aminopyridine at $262 \mathrm{~nm}$ is also observed to decrease monotonically with the growth of a new band at $233 \mathrm{~nm}$ after $5 \mathrm{~h}$, corresponding to the absorption of 4-hydroxypyridinium (Figure 1C). We can infer that the absorption band of the produced diazopyridinium cations, at $285 \mathrm{~nm}$,[32] is fully overlapped by the absorption band of residual (protonated) 4-aminopyridine by taking into account that the molar extinction coefficient for the diazopyridinium is much lower $(\varepsilon=2720$ L.mol $\left.{ }^{-1} \cdot \mathrm{cm}^{-1}\right)$ than that of the 4-amino-derivative $\left(\varepsilon=18850 \mathrm{~L} \cdot \mathrm{mol}^{-1} \cdot \mathrm{cm}^{-1}\right)$.[36] The UV-Vis spectroscopic study cannot allow the identification of all the decomposition products of the diazopyridinium cations but the results indicate that diazopyridinium cations are formed with the three aminopyridine derivatives upon in situ diazotization in aqueous acidic medium, and that they are eventually transformed into their hydrolyzed adducts.
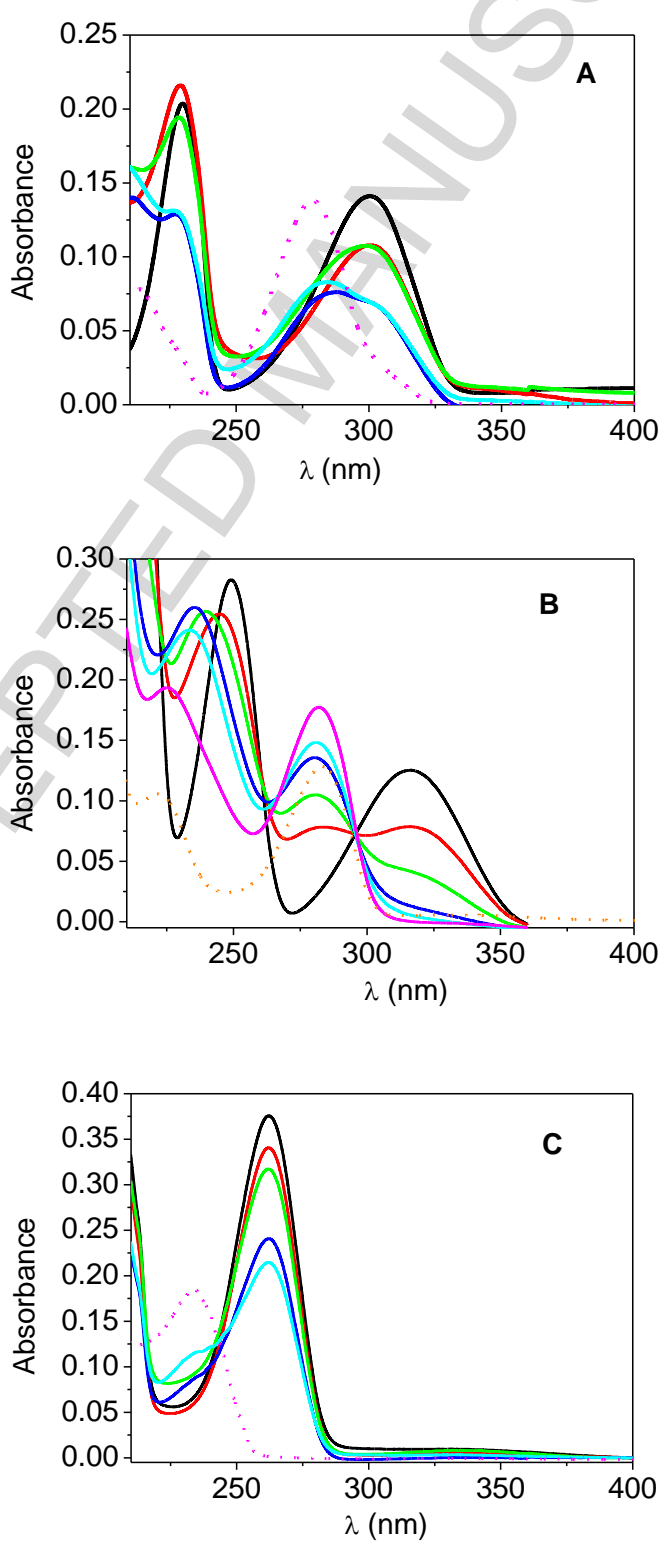
Figure 1. UV-Visible absorption spectra in $0.5 \mathrm{M} \mathrm{HCl}$ of $210^{-5} \mathrm{M}$ (A) 2-aminopyridine (black solid line) (B) 3 aminopyridine (black solid line) (C) 4-aminopyridine (black solid line). After addition of 2 eq. $\mathrm{NaNO}_{2}$, spectra were recorded at different times (A) $10 \mathrm{~min}$ (red solid line), $60 \mathrm{~min}$ (green solid line), $300 \mathrm{~min}$ (blue solid line) and $1440 \mathrm{~min}$ (cyan solid line) (B) $1 \mathrm{~min}$ (red solid line ) $5 \mathrm{~min}$ (green solid line) $10 \mathrm{~min}$ (blue solid line) $30 \mathrm{~min}$ (cyan solid line) $60 \mathrm{~min}$ (magenta solid line) (C) $10 \mathrm{~min}$ (red solid line), $60 \mathrm{~min}$ (green solid line), 300 min (blue solid line), 1440 min (cyan solid line). The absorption spectra of the corresponding hydroxypyridine compounds are also displayed for comparison purposes (dotted lines).

\subsection{Electrochemical Grafting from in situ Generated Diazopyridinium Cations.}

The electrochemical reduction of diazopyridinium cations produced from the in situ diazotization was performed at glassy carbon or gold electrodes, either by cyclic voltammetry or by chronoamperometry. Figure 2 displays a typical voltammogram obtained for the reduction of in situ produced 4-diazopyridinium cations, where the potential is swept between $+0.4 \mathrm{~V}$ and $-0.3 \mathrm{~V}$. We observed the characteristic behaviour of the electroreduction of aryl diazonium cations, with a decrease of the intensity of the cathodic current during the consecutive cycles. This behaviour corresponds to the formation of a radical species upon reduction and subsequent dediazonation. Radicals are able to graft at the electrode surface, resulting in the formation of an organic blocking layer that explains the decrease of the current intensity.[16] We have also performed the electrografting by sweeping the potential between +0.4 and $-0.8 \mathrm{~V}$ as reported by Agullo et al.[19] The blocking properties of the layers obtained with both scanning range were identical (see below and figures S1 and S2). As sodium nitrite can be competitively reduced at potential more negative than $-0.4 \mathrm{~V},[19]$ we have chosen to limit the cathodic potential incursion. 


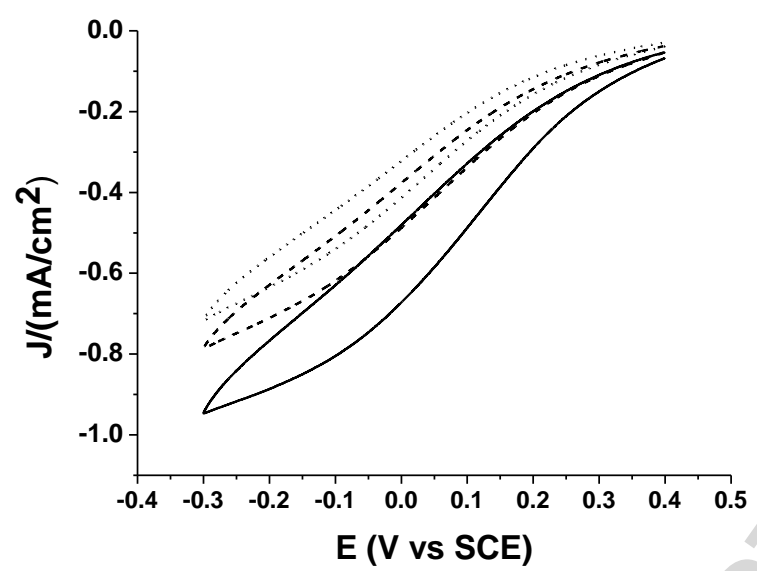

Figure 2. Recurrent cyclic voltammograms recorded at a glassy carbon electrode in an aqueous $0.5 \mathrm{M} \mathrm{HCl}$ solution containing $20 \mathrm{mM}$ of 4-aminopyridine $+40 \mathrm{mM} \mathrm{NaNO}_{2}$ after 30 s of reaction, Cycles 1 (solid line), 2 (dashed line), and 3 (dotted line) are shown. Scan rate is $0.05 \mathrm{~V} \mathrm{~s}^{-1}$

XPS measurements were performed to further assess the surface electrografting with diazopyridinium cations produced from 2-amino-, 3-amino- and 4-aminopyridine. Gold surfaces instead of carbon samples were used in order to mitigate the large contribution of the carbon atoms due to the substrate itself. The three surfaces were modified by chronoamperometry using an electrolysis time of 300s, and the reduction potential was applied $30 \mathrm{~s}$ after $\mathrm{NaNO}_{2}$ addition in the electrochemical cell. In all cases, XPS survey spectra show the presence of carbon C 1s (285 eV), oxygen $\mathrm{O} 1 \mathrm{~s}(532 \mathrm{eV}$, also present at bare gold samples, arising from contaminants) and nitrogen $\mathrm{N} 1 \mathrm{~s}(400 \mathrm{eV})$ together with intense $\mathrm{Au}$ photoelectron peaks, suggesting the formation of rather thin films. The presence of $\mathrm{N} 1 \mathrm{~s}$ photoelectron peak is a good indication of the grafting of the pyridine moieties at the surface. The N 1s core level spectra can be fitted with several components (Figure 3). 

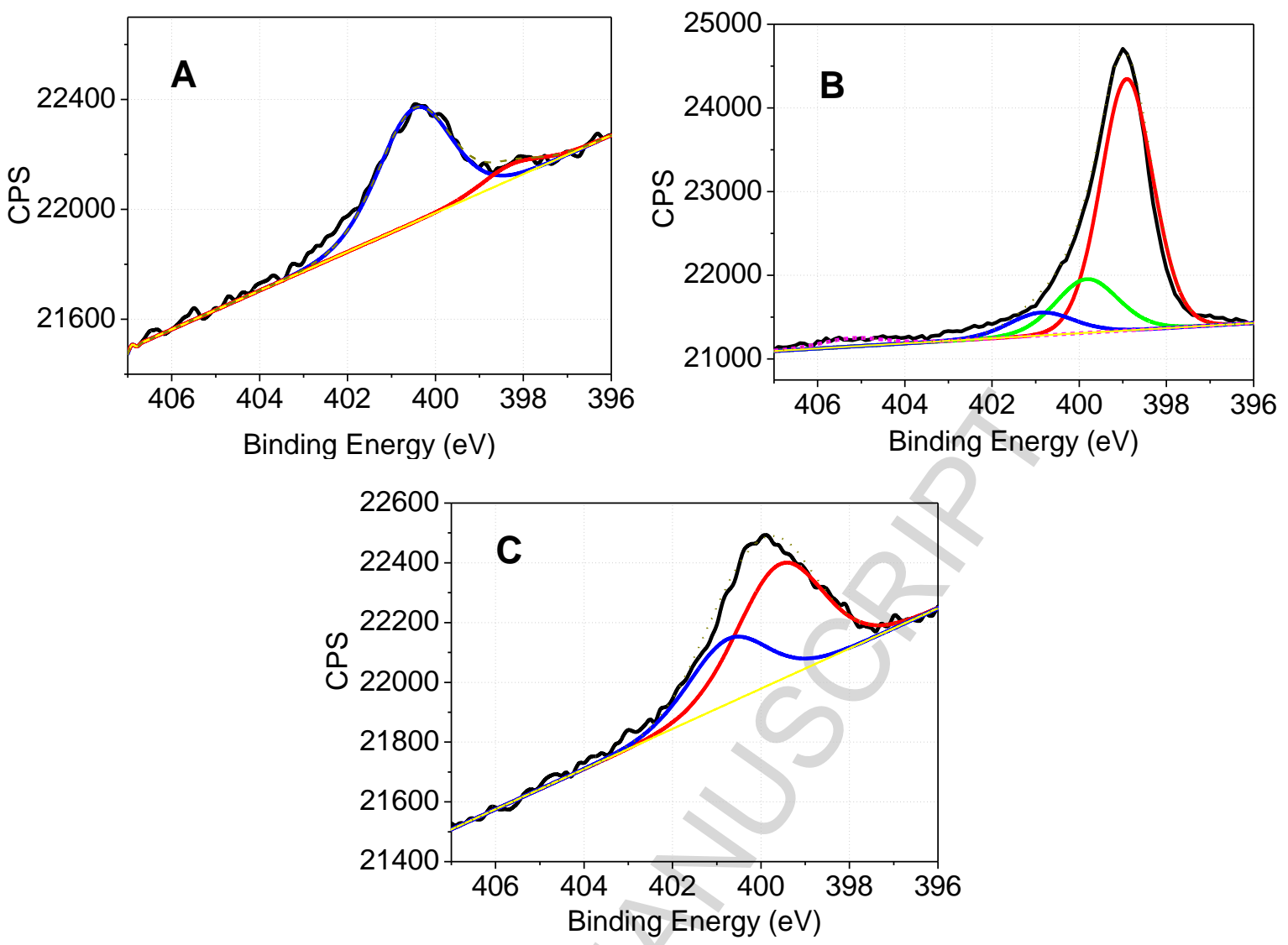

Figure 3. High resolution $\mathrm{N}$ 1s spectra for gold samples modified through electrografting of in situ generated diazopyridinium cations in $0.5 \mathrm{M} \mathrm{HCl} 30 \mathrm{~s}$ after addition of $\mathrm{NaNO}_{2}$ from (A) 2-aminopyridine, (B) 3-aminopyridine and (C) 4-aminopyridine. The experimental signal (black line) is decomposed into several components (at 398.6-399.3 eV (red curve), at 400.7$400.8 \mathrm{eV}$ (blue curve) and at $399.9 \mathrm{eV}$ (green curve, for 3-aminodiazopyridinium only) after peak-fitting.

The absence of a peak at $403.8 \mathrm{eV}$ indicates that no diazonium cations remain adsorbed at the surface. For the three samples, the contribution at $398.6-399.3 \mathrm{eV}$ is attributed to the neutral nitrogen of pyridine while the contribution at 400.7-400.8 eV corresponds to the protonated pyridine.[37] Considering the pKa of the pyridine functionality in 2-, 3- and 4-aminopyridine (6.86, 9.17 and 5.98, respectively) and the acidic grafting conditions, the nitrogen atom of the pyridine is protonated. After the electrografting process a pyridinium moiety attached to the surface is obtained, with a pKa close to that of pyridine $(\mathrm{pKa}=5.2)$. The extensive washing of the resulting surface with ultrapure water could lead to a partial neutralization of the pyridinium groups. The variation of the relative contribution of neutral nitrogen $v s$. protonated pyridine is probably due to differences in the washing procedure. An additional component at 
$399.9 \mathrm{eV}$ corresponding to azo linkages is also found in the case of electrografting from 3aminopyridine (Figure 3B), similarly to the observation made by Agullo et al.[19,20] Interestingly, this contribution is absent in the case of electrografting from the two other aminopyridine compounds. We also observed that the $\mathrm{N} 1 \mathrm{~s}$ and $\mathrm{C} 1 \mathrm{~s}$ photoelectron signals are less intense with these two compounds, relatively to the Au photoelectron signal, than those recorded in the case of 3-aminopyridine, suggesting the formation of thinner layers. Thus, the atomic ratios carbon to gold $(\mathrm{C} / \mathrm{Au})$ or nitrogen to gold (N/Au) used as quantitative parameters, are significantly larger in the case of a layer prepared with 3-aminopyridine than for the films prepared with the two other pyridine derivatives (Table 1). It can be also observed that the N/Au atomic ratio is lower for the film prepared from 2-aminopyridine (suggesting a thinner and/or less dense film) than for the film prepared from 4-aminopyridine. Ellipsometry measurements were performed to estimate the thickness of the layers onto the gold surfaces (Table 1). Because of the high roughness of the gold substrate, the thickness values cannot be considered as absolute values. Indeed, by using the scratching method with atomic force microscopy (AFM), thickness value below $1 \mathrm{~nm}$ was obtained for a film prepared with 4-aminopyridine on PPF substrates which are known to be smooth and flat substrates.[38] Nevertheless, the trend obtained onto gold surfaces show that a thicker film is obtained with 3-aminopyridine (Table 1). In this latter case, it is likely that a multilayer is formed, as reported previously,[20] and that the multilayer structure involves azo linkages as evidenced by the XPS analyses. By taking into account the evolution of the diazonium reactivity observed by UV-Visible absorption spectroscopy, films onto gold substrates were also prepared with 2- and 4-aminopyridine after $24 \mathrm{~h}$ and $5 \mathrm{~h}$ following the addition of $\mathrm{NaNO}_{2}$, respectively. XPS analyses and ellipsometry measurements give consistent results (Table 1), indicating that thin films are obtained in both cases.

Table 1. XPS atomic ratio for gold surface modified with diazopyridinium cations in situ produced from 2-,3- and 4- aminopyridine $30 \mathrm{~s}, 5 \mathrm{~h}$ or $24 \mathrm{~h}$ after addition of 2eq. of $\mathrm{NaNO}_{2}$.

\begin{tabular}{|l|l|l|l|}
\hline & $\mathrm{C} / \mathrm{Au}$ & $\mathrm{N} / \mathrm{Au}$ & $\begin{array}{c}\text { Thickness } \\
(\mathrm{nm})\end{array}$ \\
\hline 2-aminopyridine after 30s & 0.38 & 0.022 & $0.96^{\mathrm{a}}$ \\
\hline 2-aminopyridine after 24 h & 0.30 & 0.013 & $1.2^{\mathrm{a}}$ \\
\hline 3-aminopyridine after 30s & 1.11 & 0.14 & $4.1^{\mathrm{a}}$ \\
\hline 4-aminopyridine after 30s & 0.35 & 0.036 & $2.1^{\mathrm{a}}$ \\
\hline
\end{tabular}




\begin{tabular}{|l|l|l|l|}
\hline & & & $0.9 \pm 0.3^{\mathrm{b}}$ \\
\hline 4-aminopyridine after 5h & 0.33 & 0.038 & $2.3^{\mathrm{a}}$ \\
\hline
\end{tabular}

Thickness estimated by ${ }^{\text {a }}$ ellipsometry measurements of the corresponding gold surfaces.

${ }^{\mathrm{b}} \mathrm{AFM}$ scratching onto PPF substrate

\subsection{Study of the charge transfer blocking properties of the modified layers}

It is also interesting to study the blocking properties of the modified gold and carbon surfaces towards ferricyanide as a redox probe in order to indirectly demonstrate the presence of the grafted layers and to evaluate their relative amount and compactness.[39] Figure 4 shows typical voltammograms obtained with modified glassy carbon electrodes. Similar results are obtained with gold electrodes (see Figure S3). The blocking properties are easily detected through the larger peak-to-peak separation $\left(\Delta \mathrm{E}_{\mathrm{p}}\right)$ along with a decrease of the current density, because the grafted layer acts as a barrier that considerably slows down the electron transfer kinetics.
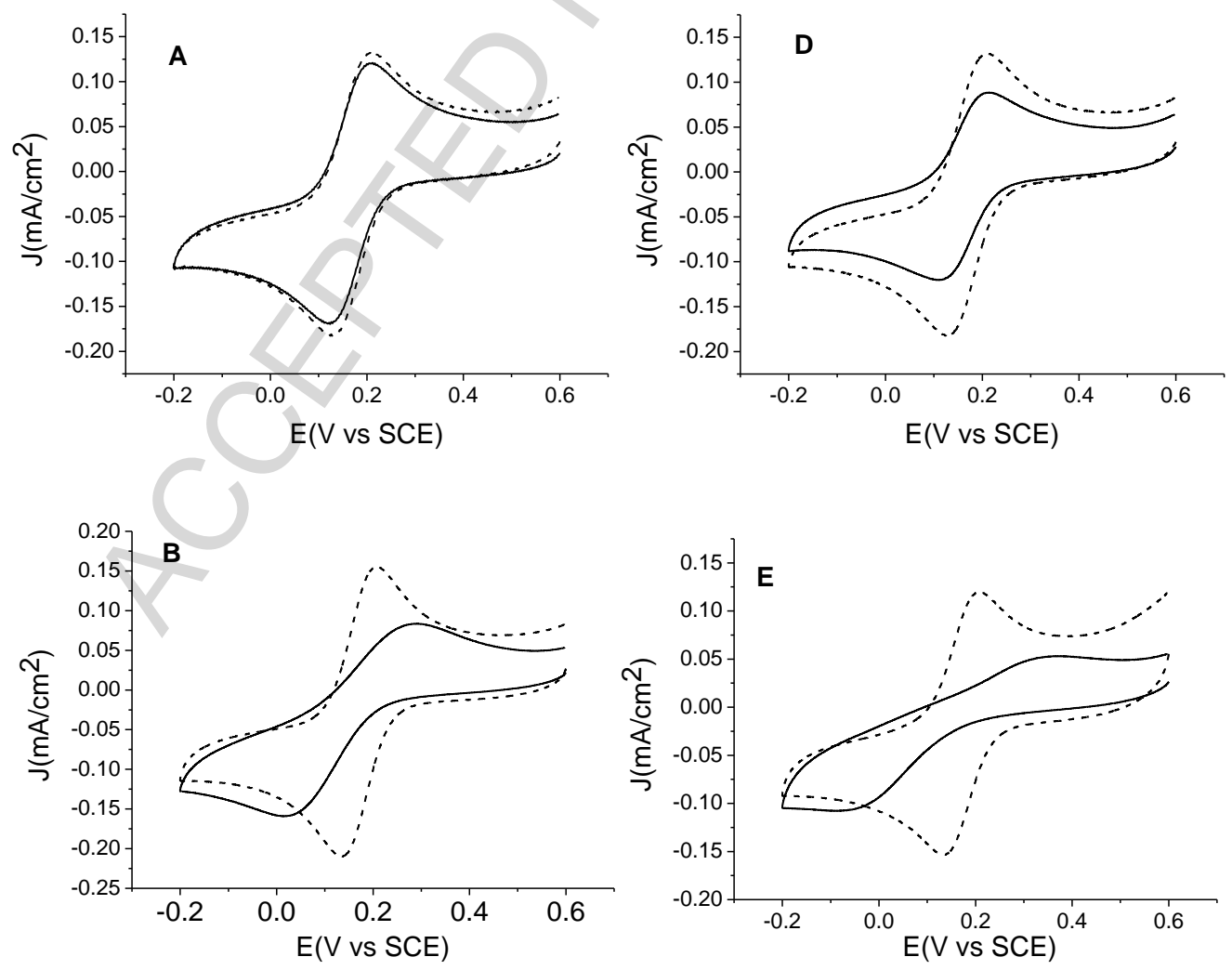

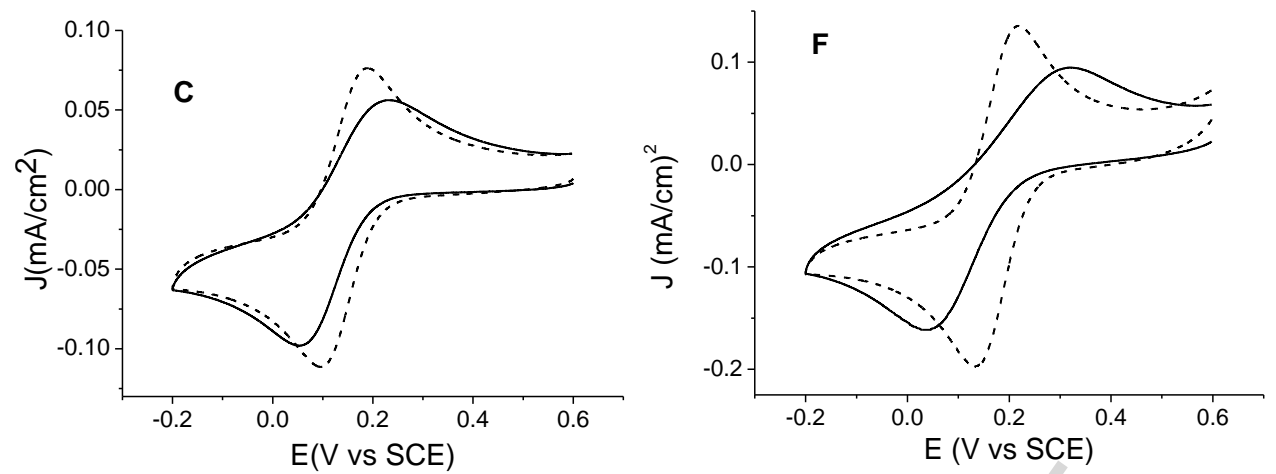

Figure 4. Cyclic voltammograms of $1 \mathrm{mM}$ ferricyanide in aqueous $0.1 \mathrm{M} \mathrm{KCl}$ solution at $\mathrm{pH}$ $=7.5$ (left column) and at $\mathrm{pH}=9$ (right column) for bare glassy carbon electrodes (dashed lines) and for modified carbon electrodes (solid lines). Modified electrodes were prepared from 2-aminopyridine (A,D), 3-aminopyridine (B,E) and 4-aminopyridine (C,F). Scan rate is $0.05 \mathrm{~V} \mathrm{~s}^{-1}$.

The blocking properties were studied at $\mathrm{pH}>\mathrm{pKa}$ of pyridine. Indeed, in aqueous acidic solution $(\mathrm{pH}=2)$, the voltammograms of modified carbon surfaces are almost identical to those of the bare surfaces because of the electrostatic interaction between the grafted pyridinium moieties and the negatively charged ferrocyanide/ferricyanide ions. At $\mathrm{pH}=7.5$ and $\mathrm{pH}=9$, a strong blocking effect is observed for films prepared with 3 -aminopyridine, this effect being more pronounced at $\mathrm{pH}=9$ because the deprotonation of the grafted pyridine is more complete in the basic aqueous solution. Accordingly, the same response to $\mathrm{pH}$ change could be observed with the electrodes modified with the two other pyridine compounds. However, the magnitude of the blocking effect strongly varies as a function of the nature of the amino-pyridine. A very poor blocking effect could be observed for the layer prepared from the 2-aminopyridine at $\mathrm{pH}=9$, with almost no significant effect at $\mathrm{pH}=7.5$ compared to the bare electrode. This result suggests the formation of thin and sparse layer onto the electrode surface, and agrees with the low value of the atomic N/Au ratio obtained from XPS data. Accordingly, the blocking character is significantly stronger in the case of layers prepared from 4-aminopyridine, although it is less marked than for the layer obtained from 3aminopyridine. This observation falls in line with the fact that thicker layers are obtained with 3-aminopyridine compared with 4-aminopyridine, as inferred by the XPS and ellipsometry measurements. Yet, the electrochemical behaviour of the redox probe at the layers prepared from 4-aminopyridine clearly indicates the formation of a thin but rather compact layer. Topography images obtained from AFM analyses onto PPF substrates further confirm that an 
homogeneous layer is formed through electrografting of diazopyridinium cations generated from 4-aminopyridine (Figure 5). The grafted PPF surface showed surface structures almost identical and with similar size to those of the bare PPF substrates, indicating a uniform deposit onto the surface. The surface roughness increased only slightly (root-mean-square roughness $\mathrm{rms}=0.4 \mathrm{~nm})$ compared to bare surface $(\mathrm{rms}=0.1 \mathrm{~nm})$, in agreement with the formation of a thin film.

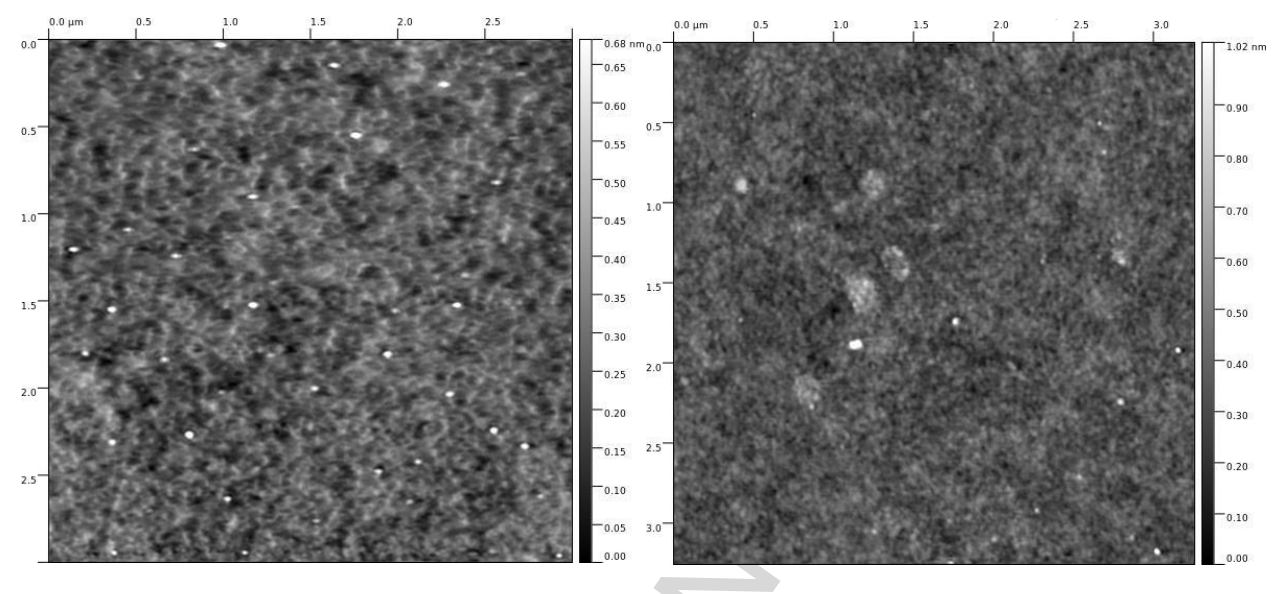

Figure 5. AFM topography images of the bare PPF substrate (left) and the modified PPF substrate from 4-aminopyridine (right).

Interestingly, the amount of charge consumed during the electrografting process (by increasing the electrolysis time) does not modify much the blocking properties of the layer (Figure S4). In principle, the aryl diazonium electrografting method allows to some extent a control of the thickness of the grafted layer by monitoring the charge density $\left(\mathrm{mC} / \mathrm{cm}^{2}\right)$ consumed during the modification process.[16] Larger charge consumption may produce thicker layer and a thicker film has generally higher blocking properties towards redox probes. This could be typically observed during the electrografting of benzenediazonium salts, in situ produced from aniline. Using $\Delta \mathrm{E}_{\mathrm{p}}$ of the ferri/ferrocyanide system as a quantifying parameter, a strong blocking effect is observed as the charge consumption is increased, reaching a full inhibition of the charge transfer process $(\Delta \mathrm{Ep}>1 \mathrm{~V})$ at these modified electrodes (Figure S5). In sharp contrast, during the electrografting of 4-diazopyridinium cations, the blocking effect is rapidly levelled and cannot be increased by extending the electrolysis time, hence with larger charge consumption,(Figure S6) suggesting that the growth of the layer might be limited to a given low to moderate thickness (self-limitation). 
Overall, the characteristics of the grafted layer obtained from the 4-aminopyridine are particularly interesting for tailoring the interface between electroactive microbial biofilms and the electrode. Indeed, it has been shown that the functionalization of electrode surface with relatively thin films provides the optimum condition relative to bacterial adhesion for improving the MFCs performance.[40]'[9]'[10] Thin grafted films are sufficient to tune the physico-chemical properties of the electrode surface while preserving fast electron transfer with electroactive species. The electrografting of 4-diazopyridinium cations hence leads to reproducible and homogeneous thin films that do not compromise fast heterogeneous electron transfer.

\subsection{Development of electroactive biofilm onto carbon surface modified from 4- aminopyridine.}

Graphite electrodes modified with films prepared with 4-aminopyridine were used for the development of electroactive biofilms, and their performance in Microbial Fuel Cells (MFC) was evaluated. In addition to their characteristics described above, the wetting properties of the resulting layer are a priori favourable for the development of electroactive biofilm notably for Geobacter-rich populations.[14] The static contact angle of a water droplet decreased from $97^{\circ}$ to $52^{\circ}$ for the PPF surface after functionalization with 4-aminopyridine, demonstrating the formation of a hydrophilic film at the PPF surface. A series of ten MFCs were fabricated by using modified electrodes with films obtained from 4-aminopyridine as anodes and compared with a series of MFCs (10 replicates) where unmodified graphite electrodes served as anodes.

Biofilm growth was monitored by measurements of the cell voltage. At the beginning of the experiment, the cell voltage is low $(100-200 \mathrm{mV})$ and after a few hours/days starts to gradually increase up to a $600-700 \mathrm{mV}$ plateau. This behaviour is consistent with the attachment, connection and growth of an electroactive biofilm onto the electrode surface. Significant differences regarding the time for the biofilm development were observed between MFCs working with modified and unmodified anodes, respectively. Faster development was systematically obtained with pyridine-modified anodes reaching their stationary phase after $46 \pm 6 \mathrm{~h}$ compared with $168 \pm 6 \mathrm{~h}$ for unmodified anodes. Examination of bioanodes samples by Scanning Electron microscopy (SEM) showed similar bacterial colonization onto electrode surfaces, indicating that the electrodes modified with 4-aminopyridine are suitable for biofilm development. 


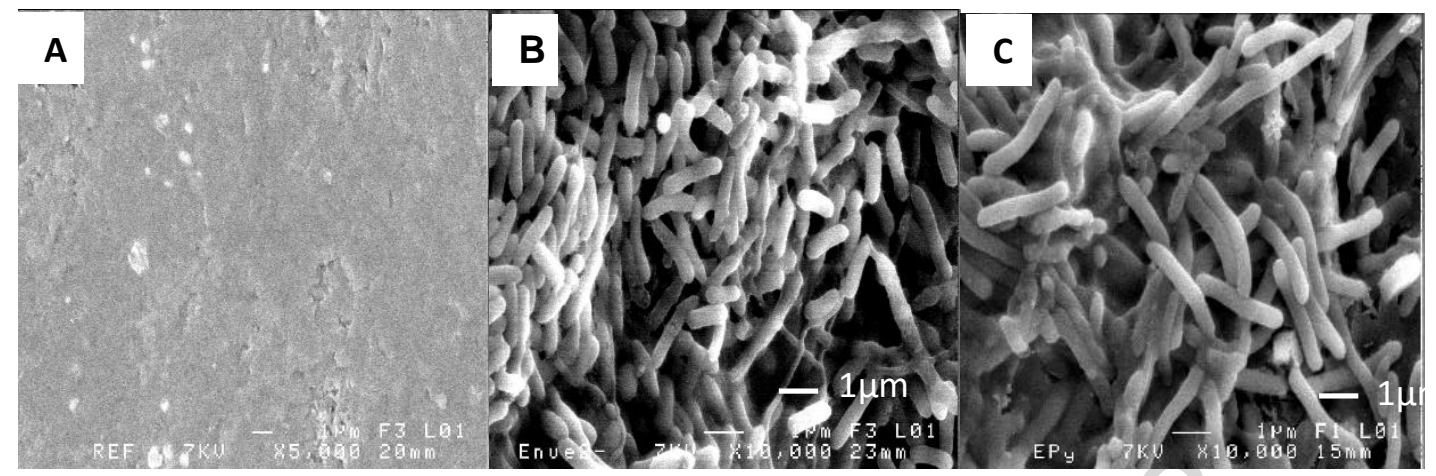

Figure 6. SEM images of (A) bare pristine graphite and (B, C) colonized graphite anodes, (B) unmodified and (C) modified with electrografting of diazopyridinium cations produced in situ from 4-aminopyridine.

Cyclic voltammetry was performed under conditions of acetate saturation $(20 \mathrm{mM})$ in order to further assess the biofilm development and to study its catalytic properties, Voltammograms recorded at slow scan rate $\left(5 \mathrm{mV} . \mathrm{s}^{-1}\right)$ exhibit the typical pattern obtained for microbial electrocatalytic anodic oxidation of acetate. The mid-point potential for the catalytic wave is around $-0.4 \mathrm{~V}$ vs SCE and corresponds to the potential of the outer-membrane c-type cytochromes responsible for the connection between the anode and acetate catabolism (Figure 7). The peculiar evolution of the electrocatalysis as a function of potential has not been totally clarified so far but has been ascribed to a combination of factors including absorption/desorption of outer-membrane c-type cytochromes at the interface, metabolic gated mechanism at high overpotentials and/or the involvement of different outer-membrane c-type cytochromes with different potential and different responses to high overpotentials. The MFCs with modified anodes had higher performances than those with the unmodified surfaces. MFC working with graphite surface grafted with layer obtained from 4aminopyridine displayed a maximum catalytic current density of $0.100 \pm 0.02 \mathrm{~mA} . \mathrm{cm}^{-2}$, which is 4 fold higher than for the bare graphite electrode $\left(0.02 \pm 0.01 \mathrm{~mA} \cdot \mathrm{cm}^{-2}\right)$. 


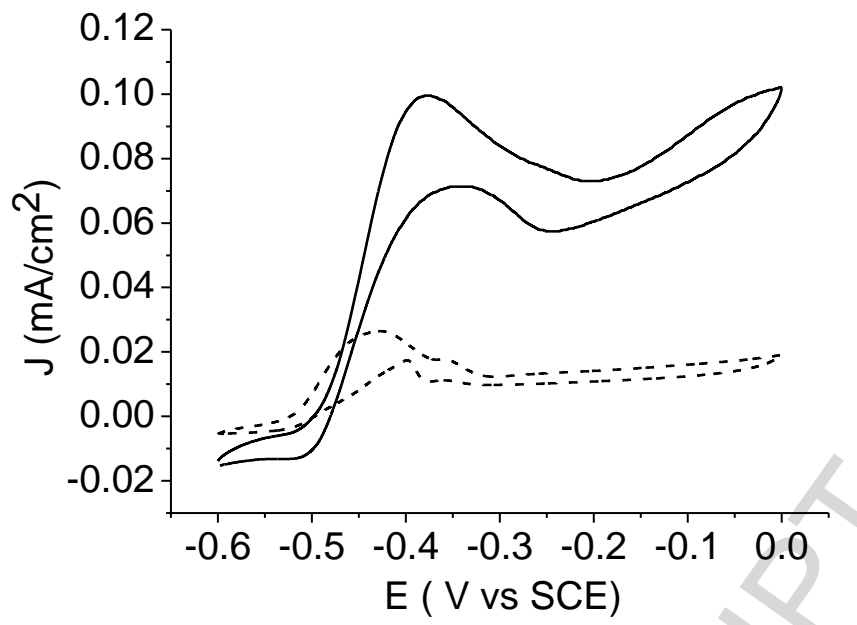

Figure 7. Cyclic voltammograms showing acetate biocatalytic oxidation at acetate saturation conditions $(20 \mathrm{mM})$ onto graphite bioanodes. Biofilms (one week development after reaching the stationary phase) were formed on bare surface (dashed line) and on modified surface with 4-aminopyridine (solid line). Scan rate is $5 \mathrm{mV} \mathrm{s}^{-1}$.

As control experiments, a series of MFCs using bare graphite electrodes immerged in an anolyte that contains $0.1 \mathrm{mM}$ of pyridine were also studied. In this case, the biofilm was developed after ca. 2 days. The catalytic properties were similar to those observed with the grafted anodes, with a maximum catalytic current density equal to $0.09 \mathrm{~mA} . \mathrm{cm}^{-2}$ (Figure 7). These observations clearly indicate that pyridine has a beneficial effect on the biofilm development and catalytic efficiency. However, when pyridine is containing in the anolyte as a freely diffusing species, the catalytic signal rapidly decreases to $0.015 \mathrm{~mA} . \mathrm{cm}^{-2}$ after the biofilm was developed (Figure S8). In contrast, MFCs involving the grafted pyridine onto the anode continue to perform at higher current densities for several weeks.

Chronoamperometry measurements at maximal catalytic activity $(E=-0.4 \mathrm{~V}$ vs SCE) were recorded, allowing the monitoring of the Faradic efficiency. $2 \mathrm{mM}$ of acetate was added at the beginning of each experiment. The current was recorded at constant temperature at functional bioanodes aged of one week (bare and modified graphite surfaces) until full acetate consumption. The chronoamperometric curves are shown in Figure 8 and further confirm the better performance of the bioanodes obtained from the modified surface. 


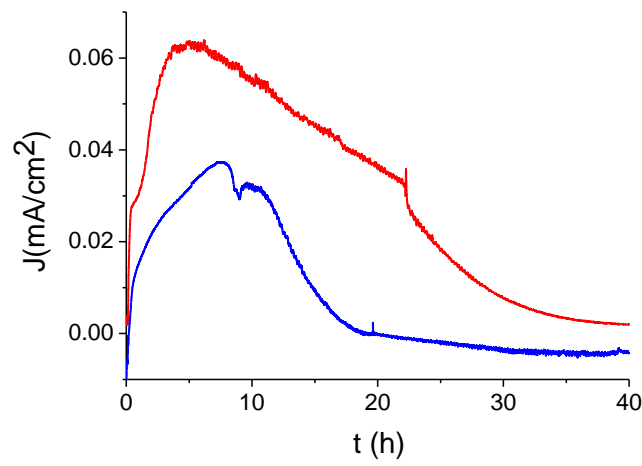

Figure 8. Chronoamperometry curves recorded at maximum catalytic current density (applying a potential $\mathrm{E}=-0.4 \mathrm{~V}$ vs $\mathrm{SCE}$ ) after addition of $2 \mathrm{mM}$ of potassium acetate for biofilms developed after one week either on bare graphite electrode (blue line), or on surface modified electrode (red line).

The faradic efficiency of the MFC could be calculated from these curves (Table 2) and the obtained values are consistent with previous works.[41] The highest efficiency (40\%) was found for the bioanodes where biofilm were grown onto modified surface. Note that the efficiency drops to $3 \%$ for MFC with $0.1 \mathrm{mM}$ pyridine as a freely diffusing species in the anolyte.

Table 2. Faradic efficiency of MFC involving different bioanodes colonized by biofilms after one week of development

\begin{tabular}{|c|c|}
\hline Biofilms developed after one week & $\begin{array}{c}\text { Faradic } \\
\text { efficiency \% }\end{array}$ \\
\hline Bare graphite & 10 \\
\hline Modified graphite with layers prepared from 4-aminopyridine & 40 \\
\hline Bare graphite with anolyte containing 0.1 mM pyridine & 3 \\
\hline
\end{tabular}

The surface modification obviously improves the biofilm development and connection to the electrode. The nature of the grafted moiety (pyridine) is likely to play a role in the improvement of the biofilm development and catalytic properties, as suggested by the control experiment with freely-diffusing pyridine. Explanation for this beneficial effect probably lies 
in a combination of different effects including the hydrophilic character of the layer, the formation of a thin film and the presence of positive charges within the layer (partial protonation of pyridine units, strengthened by the acidification of the biofilm). Finally, possible favourable specific interactions with the c-type outer membrane cyctochromes cannot be excluded since pyridine ligands can coordinate the metal centre of the heme group of c-type cytochromes.[26]'[27]

\section{Conclusions}

Carbon electrodes were functionalized by the electrochemical reduction of diazopyridinium cations produced in situ in acidic aqueous solution from 2-, 3- and 4-aminopyridine. XPS and electrochemical analyses confirmed the presence of immobilized pyridine units. The reactivity versus diazotization of the aminopyridine isomers is different: 3-aminopyridine allows the formation of compact and thicker layers than 2-and 4-aminopyridine. While 2-aminopyridine leads to sparse and thin layer, it is possible to prepare thin but homogeneous and more compact film with 4-aminopyridine where the thickness growth is limited by the reactivity of the resulting cations. The grafting of thin and homogeneous hydrophilic films that do not block electron transfer under simple and reproducible experimental conditions is particularly advantageous for the tailoring of the interface between the electrode surface and a bacterial anodic biofilm. Graphite electrodes modified through the electrografting of 4-diazopyridinium cations were implemented as anodes in fuel cells containing wastewater and acetate as the organic substrate for the bacteria. Electroactive biofilms are successfully and more rapidly grown onto the pyridine-grafted electrodes. They present better catalytic performance toward acetate oxidation than those developed onto bare graphite electrodes.

\section{Experimental Section}

Chemicals. 2-aminopyridine (99 \%), 3-aminopyridine (99 \%), 4-aminopyridine (99 \%), 3hydroxypyridine and 4-hydroxypyridine were purchased from Alfa Aesar and used without further purification. 2-hydroxypyridine was obtained from Acros. Potassium hydrogen phosphate and potassium dihydrogen phosphate (Alfa Aesar) were used to prepare phosphate buffer solutions. Potassium ferricyanide was purchased from Acros. Sodium nitrite and hydrochloric acid (37\%) were obtained from Sigma-Aldrich. All solutions were prepared with 
ultrapure water $(18.2 \mathrm{M} \Omega \mathrm{cm})$, thoroughly degassed under argon during 20 min prior to experiment.

Surface modifications procedures. Different materials were used as electrodes, considering the specific requirements of the different characterization techniques used. For electrochemical analyses, glassy carbon or gold disk electrodes were employed. They were obtained from IJ Cambria ( $3 \mathrm{~mm}$ and $1.8 \mathrm{~mm}$ diameter, respectively). Gold plates or homemade PPF (pyrolized photoresist film) substrates of approximately $1 \mathrm{~cm}^{2}$ were employed for ellipsometry, XPS and AFM measurements, respectively. PPF substrates exhibit a low roughness that is suitable for AFM measurements while gold substrates allow clean ellipsometry measurements (requiring a reflective surface) and XPS analyses (allowing mitigation of the C1s photoelectron signal) (see text). Gold plates (gold layer of $100 \mathrm{~nm}$ thickness onto Si wafer substrate) were purchased from Sigma-Aldrich and cleaned before surface functionalization (piranha solution). Caution! Piranha solution is a very strong oxidant and is extremely dangerous to work with; it should be handled very carefully. Graphite electrodes with large surface area were employed in the MFCs, these materials being more cost-effective for such applications compared to glassy carbon material. A platinum gauze and saturated calomel electrode (SCE) were used as counter and reference electrodes respectively. The glassy carbon or Au disk electrodes were polished using SiC paper (Struers, grain size 4000). After each polishing the electrode was rinsed with ultrapure water and kept in water.

The electrodes were modified by cyclic voltammetry or chronoamperometry using a $20 \mathrm{mM}$ diazonium cations solution in acid medium $(0.5 \mathrm{M} \mathrm{HCl})$. In order to generate in situ diazonium cations, $40 \mathrm{mM} \mathrm{NaNO}_{2}$ was added to $20 \mathrm{mM}$ aminopyridine precursors in $0.5 \mathrm{M}$ $\mathrm{HCl}$ solution under vigorous stirring at 0 to $5{ }^{\circ} \mathrm{C} .30 \mathrm{~s}, 5 \mathrm{~h}$ or $24 \mathrm{~h}$ after the $\mathrm{NaNO}_{2}$ addition, the electrode was immersed into the mixture solution. A potential of $+0.4 \mathrm{~V}$ was applied for $10 \mathrm{~s}$, and thereafter the electrode was cycled 3 times between +0.4 and $-0.3 \mathrm{~V}$ at $50 \mathrm{mV} \mathrm{s}^{-1}$ (stirring is turned off a few seconds before the electrochemical experiment). Alternatively, the potential is hold at $-0.25 \mathrm{~V}$ or $-0.35 \mathrm{~V}$ for $300 \mathrm{~s}$ for achieving surface modification from 4aminopyridine and 2- or 3-aminopyridine, respectively. The final potential in cyclic voltammetry or the applied potential in chronoamperometry are sufficiently negative for the electrografting process to be controlled by the mass transfer. Electrodes were then abundantly rinsed with ultrapure water. 
Microbial Fuel Cells. The electrodes consist of graphite pieces of 5-4 cm x 1.4-1.5 $\mathrm{cm}$ x 0.4$0.5 \mathrm{~cm}$ polished with grit $500 \mathrm{SiC}$ papers (Struers), before direct integration in biofuel cells or prior to modification with diazopyridinium cations. Fuel cells were built using an " $\mathrm{H}$ " type reactor configuration as described in [42]. The microbial inoculum was collected from the Beaurade Wastewater Treatment Plant, Rennes (France). The anode compartment was filled with $200 \mathrm{~mL}$ of wastewater and $50 \mathrm{~mL}$ of $0.1 \mathrm{M}$ phosphate buffer $(20 \mathrm{mM}$ final concentration) and fed with $20 \mathrm{mM}$ (final concentration in the anolyte) of sodium acetate (addition of $2.5 \mathrm{~mL}$ of a $2 \mathrm{M}$ stock solution in phosphate buffer). The anodic compartment was covered with aluminium foil as protection against sunlight. The $\mathrm{pH}$ of the anolyte was kept at $\mathrm{pH}=7.5$. The $\mathrm{pH}$ is measured every week and adjusted if needed by addition of small volumes $(<500 \mu \mathrm{L})$ of concentrated $\mathrm{HCl}$ or $\mathrm{NaOH}$ solutions. The anolyte was thoroughly degassed by argon bubbling at the beginning of the experiments and all the ports were tightly plugged. All solutions were thoroughly degassed prior to addition. Thus, experiments are performed under anaerobic conditions. A $0.1 \mathrm{M}$ potassium ferricyanide in a $20 \mathrm{mM}$ phosphate buffer solution was used as electron acceptor at the cathode side in order to ensure a stable cathode potential and a non-limiting current. The two cell compartments were separated through an ion exchange membrane (Fumasep® FTCM-E Polyethylene Terephtalate (PET)reinforced, Fumatech, Germany). The electrodes were connected through a $1 \mathrm{k} \Omega$ external resistance. Reactors were kept at $25-30^{\circ} \mathrm{C}$. Cell voltages were measured using a digital multimeter (Velleman DVM9912).

Instrumentation. Electrochemical experiments were carried out with an Autolab electrochemical analyser (PGSTAT302N galvanostat/potentiostat from Eco Chemie B.V.) in a three-electrode set-up.

AFM experiments used a NT-DMT Ntegra microscope. The topography images were acquired in acoustic mode using silicon nitride tips (resonance frequency ca $350 \mathrm{kHz}$ ). The scratching technique was used to determine the thickness of the organic layer grafted on the PPF samples. Square-shaped scratches were obtained in contact mode with a force set-point voltage around $1 \mathrm{~V}$. Several depth-profiles were measured for each scratched region. Height distributions were also investigated on images to confirm the depth-profiles measurements.

The static contact angles of $2 \mu \mathrm{L}$ ultrapure water drops were measured on four different spots on the surface with an easy drop goniometer (Krüss). The contact angles were determined using a tangent 2 or circle fitting models. 
Thicknesses of the layers on Au were measured with a spectroscopic ellipsometer $\alpha$-SE (J.A. Woollam. Co.). The polarization angles $\Psi$ and $\Delta$ were recorded in the $300-900 \mathrm{~nm}$ wavelength range at an incident angle of $70^{\circ}$. The optical constants were fitted by assuming $\mathrm{n}=1.5$ (refraction index) and $\mathrm{k}=0$ (extinction coefficient) for the organic layer while $\mathrm{n}_{\mathrm{s}}=0.203$ and $\mathrm{k}_{\mathrm{s}}=3.431$ were taken for gold. Measurements reported are the average of ten data points across each substrate.

UV-Visible absorption spectra were recorded with a Shimadzu UV-1605 spectrophotometer employing quartz cuvette with $10 \mathrm{~mm}$ optical path length.

X-ray photoelectron spectroscopy data have been collected using a Kratos Axis Nova spectrometer using the $\mathrm{Al} \mathrm{K \alpha} \mathrm{X-ray} \mathrm{source} \mathrm{working} \mathrm{at} 1486.6 \mathrm{eV}$ and using a spot size of $0.7 \times 0.3 \mathrm{~mm}^{2}$. Survey spectra $(0-1000 \mathrm{eV})$ were acquired with an analyzer pass energy of 160 $\mathrm{eV}(0.5 \mathrm{eV} / \mathrm{step})$; high resolution spectra used a pass energy of $40 \mathrm{eV}(0.1 \mathrm{eV} / \mathrm{step})$. Binding energies were referenced to $\mathrm{C} 1 \mathrm{~s}$ peak at $285 \mathrm{eV}$. The core level spectra were peak-fitted using the CasaXPS software, Ltd., version 2.3.18).

Scanning Electron Microscopy (SEM) analyses were performed with JSM-6301F (Jeol) scanning electron microscope (Scan-MAT CMEBA, Rennes). The SEM microscope is equipped with a field-emission gun working under vacuum condition at $10^{-9}$ Torr. Electron beam was accelerated through a voltage of $7 \mathrm{kV}$ and $15 \AA$. Samples were fixed overnight with a $2.5 \%$ glutaraldehyde phosphate buffer solution $(0.1 \mathrm{M}, \mathrm{pH}=7)$ at room temperature. Then, they were thoroughly washed in aqueous solutions containing 60, 70, 80, $90 \%$ ethanol. A final washing in $100 \%$ ethanol was performed and samples were critically-point dried, then coated with Au prior to SEM experiments.

\section{Acknowledgements}

The authors are grateful to F. Gouttefangeas and L. Joanny for the SEM measurements (ScanMAT (UMS CNRS University of Rennes 1), CMEBA). J. Hamon (Institut des Matériaux de Nantes, France) is thanked for his help in recording the XPS spectra. Dr. M. Cortés is thanked for help in some of the microbial fuel cells experiments. H.Smida thanks the French Ministry of Research for her grant. E. Lebègue thanks the EU Marie Skłodowska Curie Individual Fellowship action for financial support. 
[1] H. Smida, T. Flinois, E. Lebègue, C. Lagrost, F. Barrière "Microbial Fuel CellsWastewater Utilization" in Reference Module in Chemistry, Molecular Sciences and Chemical Engineering,Encyclopedia of Interfacial Chemistry: Surface Science and Electrochemistry; K. Vandelt \& P. Vagdama, Ed.; Elsevier, 2017.

[2] A. P. Borole, G. Reguera, B. Ringeisen, Z.-W. Wang, Y. Feng, B. H. Kim. Electroactive biofilms: Current status and future research needs. Energy Environ. Sci. 4 (2011) 4813-4834.

[3] T. Nam, S. Son, B. Koo, H. V. Hoa Tran, J. R. Kim, Y. Choi, S. P. Jung. Comparative evaluation of performance and electrochemistry of microbial fuel cells with different anode structures and materials. Int. J. Hydrog. Energy, 42 (2017), 27677-27684. Cercado, L. F. Cházaro-Ruiz, V. Ruiz, I. d. J. López-Prieto, G. Buitrón, E. RazoFlores. Biotic and abiotic characterization of bioanodes formed on oxidized carbon electrodes as a basis to predict their performance. Biosens. Bioelectron. 50 (2013) 373-381.

[5] B. Li, J. Zhou, X. Zhou, X. Wang, B. Li, C. Santoro, M. Grattieri, S. Babanova, K. Artyushkova, P. Atanassovet al. Surface Modification of Microbial Fuel Cells Anodes: Approaches to Practical Design. Electrochim. Acta 134 (2014) 116-126.

[6] J. Du, C. Catania, G. C. Bazan. Modification of Abiotic-Biotic Interfaces with Small Molecules and Nanomaterials for Improved Bioelectronics. Chem. Mater. 26 (2014) 686-697.

[7] G. G. kumar, V. G. S. Sarathi, K. S. Nahm. Recent advances and challenges in the anode architecture and their modifications for the applications of microbial fuel cells. Biosens. Bioelectron. 43 (2013) 461-475.

[8] K. Guo, A. Prévoteau, S. A. Patil, K. Rabaey. Engineering electrodes for microbial electrocatalysis. Curr. Op. Biotechnol. 33 (2015) 149-156.

[9] T. Saito, M. Mehanna, X. Wang, R. D. Cusick, Y. Feng, M. A. Hickner, B. E. Logan. Effect of nitrogen addition on the performance of microbial fuel cell anodes. Biores. Technol. 102 (2011) 395-398.

[10] M. Picot, L. Lapinsonnière, M. Rothballer, F. Barrière. Graphite anode surface modification with controlled reduction of specific aryl diazonium salts for improved microbial fuel cells power output. Biosens. Bioelectron. 28 (2011) 181-188.

[11] L. Lapinsonnière, M. Picot, F. Barrière. Enzymatic versus Microbial Bio-Catalyzed Electrodes in Bio-Electrochemical Systems. ChemSusChem 5 (2012) 995-1005.

[12] L. Lapinsonnière, M. Picot, C. Poriel, F. Barrière. Phenylboronic Acid Modified Anodes Promote Faster Biofilm Adhesion and Increase Microbial Fuel Cell Performances. Electroanalysis 25 (2013) 601-605.

[13] A. Kumar, P. O. Conghaile, K. Katuri, P. Lens, D. Leech. Arylamine functionalization of carbon anodes for improved microbial electrocatalysis. RSC Adv. 3 (2013) 1875918761.

[14] K. Guo, S. Freguia, P. G. Dennis, X. Chen, B. C. Donose, J. Keller, J. J. Gooding, K. Rabaey. Effects of Surface Charge and Hydrophobicity on Anodic Biofilm Formation, Community Composition, and Current Generation in Bioelectrochemical Systems. Environ. Sci. Technol. 47 (2013) 7563-7570.

[15] A. S. Commault, F. Barrière, L. Lapinsonnière, G. Lear, S. Bouvier, R. J. Weld. Influence of inoculum and anode surface properties on the selection of Geobacterdominated biofilms. Biores. Technol. 195 (2015) 265-272.

[16] D. Belanger, J. Pinson. Electrografting: a powerful method for surface modification. Chem. Soc. Rev. 40 (2011) 3995-4048. 
[17] T. Breton, A. J. Downard. Controlling Grafting from Aryldiazonium Salts: A Review of Methods for the Preparation of Monolayers. Aust. J. Chem. 70 (2017) 960-972.

[18] R. N. Butler. Diazotization of heterocyclic primary amines. Chem. Rev. 75 (1975) 241-257.

[19] J. Agullo, M. Morin, D. Bélanger. Modification of Glassy Carbon Electrode by Electrografting of In Situ Generated 3-diazopyridinium Cations. J. Electrochem. Soc. 159 (2012) H758-H764.

[20] J. Agullo, S. Canesi, F. Schaper, M. Morin, D. Bélanger. Formation and Reactivity of 3-Diazopyridinium Cations and Influence on Their Reductive Electrografting on Glassy Carbon. Langmuir 28 (2012) 4889-4895.

[21] Q. Li, K. Schönleber, P. Zeller, I. Höhlein, B. Rieger, J. Wintterlin, K. Krischer. Activation of silicon surfaces for $\mathrm{H} 2$ evolution by electrografting of pyridine molecules. Surf. Sci. 631 (2015) 185-189.

[22] H. Leinonen, M. Lajunen. Fascinating distinct reactivity of 3- or 2-aminopyridines with carbon nanotubes. Chem. Eng. J. 172 (2011) 557-563.

[23] A. Yeşildağ, D. Ekinci. Covalent attachment of pyridine-type molecules to glassy carbon surfaces by electrochemical reduction of in situ generated diazonium salts. Formation of ruthenium complexes on ligand-modified surfaces. Electrochim. Acta 55 (2010) 7000-7009.

[24] M. K. Bayazit, L. S. Clarke, K. S. Coleman, N. Clarke. Pyridine-Functionalized Single-Walled Carbon Nanotubes as Gelators for Poly(acrylic acid) Hydrogels. J. Am. Chem. Soc. 132 (2010) 15814-15819.

[25] G. Yang, L. Wang, J. Jia, D. Zhou, D. Li. Chemically modified glassy carbon electrode for electrochemical sensing paracetamol in acidic solution. J. Solid-State Electrochem. 16 (2012) 2967-2977.

[26] M. Fedurco. Redox reactions of heme-containing metalloproteins: dynamic effects of self-assembled monolayers on thermodynamics and kinetics of cytochrome c electrontransfer reactions. Coord. Chem. Rev. 209 (2000) 263-331.

[27] M. J. Eddowes, H. A. O. Hill. Electrochemistry of horse heart cytochrome c. J. Am. Chem. Soc. 101 (1979) 4461-4464.

[28] A. Kumar, L. H.-H. Hsu, P. Kavanagh, F. Barrière, P. N. L. Lens, L. Lapinsonnière, J. H. Lienhard V, U. Schröder, X. Jiang, D. Leech. The ins and outs of microorganismelectrode electron transfer reactions. Nat. Rev. Chem. 1 (2017) 0024.

[29] R. Kumar, L. Singh, Z. A. Wahid, M. F. M. Din. Exoelectrogens in microbial fuel cells toward bioelectricity generation: a review. Int. J. Energy Res. 39 (2015) 10481067.

[30] D. R. Bond, D. E. Holmes, L. M. Tender, D. R. Lovley. Electrode-Reducing Microorganisms That Harvest Energy from Marine Sediments. Science 295 (2002) 483.

[31] D. R. Lovley. The microbe electric: conversion of organic matter to electricity. Curr. Op. Biotechnol. 19 (2008) 564-571.

[32] E. Kalatzis. Reactions of N-heteroaromatic bases with nitrous acid. Part I. Diazotisation and nitrosation of [small alpha]- and [gamma]-amino-derivatives in dilute acid solutions. J. Chem. Soc. B, DOI:10.1039/J29670000273 10.1039/J29670000273 (1967) 273-277.

[33] E. Kalatzis. Reactions of N-heteroaromatic bases with nitrous acid. Part II. The kinetics of the diazotisation of 4-aminopyridine. J. Chem. Soc. B, DOI:10.1039/J29670000277 10.1039/J29670000277 (1967) 277-282. 
[34] E. Kalatzis, C. Mastrokalos. Reactions of N-heteroaromatic bases with nitrous acid. Part III. Kinetics of diazotisation of 2-aminopyridine. J. Chem. Soc. Perkin Trans 2, DOI:10.1039/P29740000498 10.1039/P29740000498 (1974) 498-502.

[35] E. A. Steck, G. W. Ewing, F. Nachod. Absorption Spectra of Heterocyclic Compounds. II. Amino-Derivatives of Pyridine, Quinoline and Isoquinoline. J. Am. Chem. Soc. 70 (1948) 3397-3406.

[36] C. A. Bunton, M. J. Minch, B. B. Wolfe. Decomposition of pyridine-2- and -4diazotates. J. Am. Chem. Soc. 96 (1974) 3267-3275.

[37] D. N. Hendrickson, J. M. Hollander, W. L. Jolly. Nitrogen ls electron binding energies. Correlations with molecular orbital calculated nitrogen charges. Inorg. Chem. 8 (1969) 2642-2647.

[38] F. Anariba, S. H. DuVall, R. L. McCreery. Mono- and Multilayer Formation by Diazonium Reduction on Carbon Surfaces Monitored with Atomic Force Microscopy "Scratching". Anal. Chem. 75 (2003) 3837-3844.

[39] M. D'Amour, D. Bélanger. Stability of Substituted Phenyl Groups Electrochemically Grafted at Carbon Electrode Surface. J. Phys. Chem. B 107 (2003) 4811-4817.

[40] A. Terada, A. Yuasa, T. Kushimoto, S. Tsuneda, A. Katakai, M. Tamada. Bacterial adhesion to and viability on positively charged polymer surfaces. Microbiol. 152 (2006) 3575-3583.

[41] L. Huang, B. E. Logan. Electricity production from xylose in fed-batch and continuous-flow microbial fuel cells. Appl. Microbiol. Biotechnol. 80 (2008) 655.

[42] O. Schaetzle, F. Barriere, U. Schroder. An improved microbial fuel cell with laccase as the oxygen reduction catalyst. Energy Environ. Sci. 2 (2009) 96-99. 


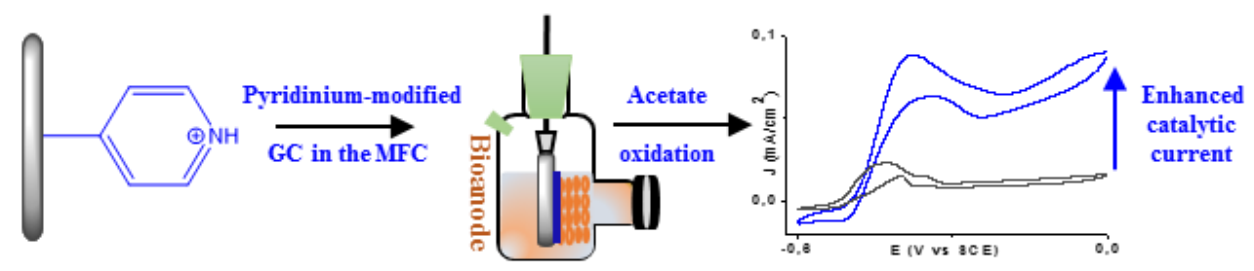

Graphical abstract 


\section{Highlights}

- Electrografting of in situ produced 3 diazopyridinium isomer cations onto carbon surface

- Efficient tailoring of the interface between anode and a bacterial biofilm

- Grafted Pyridine units promote faster biofilm development 